\title{
Propagation features of a class of beams radiated from pseudo-Schell model vectorial sources
}

\author{
Rosario Martínez-Herrero ${ }^{1, *}$, Gemma Piquero $^{1}$, Juan Carlos González de Sande ${ }^{2}$, Massimo Santarsiero $^{3}$, and \\ Franco Gori $^{3}$ \\ ${ }^{1}$ Departamento de Óptica, Universidad Complutense de Madrid, Ciudad Universitaria, 28040 Madrid, Spain; \\ ${ }^{2}$ Dipartimento di Ingegneria, Università Roma Tre, Via V. Volterra 62, 00146 Rome, Italy; \\ ${ }^{3}$ ETSIS de Telecomunicación, Universidad Politécnica de Madrid, Campus Sur 28031 Madrid, Spain;
}

\begin{abstract}
Pseudo Schell-model sources were introduced and studied in the scalar domain as those partially coherent sources that present a degree of coherence depending on the difference between the radial coordinates of the two considered points. In this work we study the propagation features of a class of beams radiated from sources of this kind, but endowed with a vectorial nature. The polarization pattern and the degree of polarization are not uniform across the beam section but remain invariant upon free paraxial propagation. On the other hand, their coherence and irradiance features are also non-uniform but in this case they change at each $z$-plane. The field characteristics can be varied on acting on the free parameters of the cross-spectral density matrix of the source.
\end{abstract}

\section{Introduction}

The proposal of new types of light beams with structured coherence and polarization is a subject of increasing recent research in the literature [1-8]. The interest for such beams lies in the peculiar properties that they may present, both across the source plane and upon propagation. Just to quote some of them, we recall the self-focusing, particle trapping, or propagation through turbulent media [9-15]

A recently introduced example of structured sources in the scalar realm are the so-called pseudoSchell model (PSM) sources [7, 8], which present coherence characteristics that only depend on the difference of the radial distance to the center of two points in the source transverse section, i.e., on the difference of their radial coordinates. Vectorial PSM (VPSM) sources have also been proposed by Hyde [16] who, in addition, presented a scheme aimed at synthesizing them.

In this work we focus our attention onto a class of vectorial VPSM sources, whose polarization characteristics only depend on the azimuthal coordinate across the source plane, and study the coherence and polarization features of the radiated beams in the paraxial regime. The proposed sources present a degree of polarization dependent on the point across the transverse section of the source, in particular on the azimuthal coordinate. The intensity at the source plane has a null at the center and the coherence characteristics only depend on the difference between the radial coordinates of the two considered points. An

*e-mail: r.m-h@fis.ucm.es interesting feature of these sources is that the polarization characteristics remain invariant upon free propagation being useful, for example, in polarimetry [17].

\section{Source model}

The VPSM source we are going to study is characterized by a cross-spectral density matrix (CSDM) across the source plane of the following form:

$$
\widehat{W}\left(\mathbf{r}_{1}, \mathbf{r}_{2}\right)=W_{\mathrm{PS}}\left(r_{1}, r_{2}\right) \widehat{H}\left(\theta_{1}, \theta_{2}\right),
$$

where $\mathbf{r}_{j}=\left(r_{j}, \theta_{j}\right)$ (with $j=1,2$ ) are the polar coordinates of two points across the source plane. $W_{P S}\left(r_{1}, r_{2}\right)$ is a scalar cross-spectral density (CSD) of the Gaussian pseudo-Schell model type [7, 8], i.e.,

$$
W_{\mathrm{PS}}\left(r_{1}, r_{2}\right)=I r_{1} r_{2} \exp \left[-\frac{r_{1}^{2}+r_{2}^{2}}{w_{0}^{2}}-\frac{\left(r_{1}-r_{2}\right)^{2}}{\delta^{2}}\right]
$$

being $I$ a real positive constant, and $w_{0}$ and $\delta$ two distances related the intensity width and the spatial coherence of the source, respectively. Furthemore, $\widehat{H}\left(\theta_{1}, \theta_{2}\right)$ is a $2 \times 2$ matrix having components

$$
\begin{aligned}
& H_{x x}=\cos \left(\theta_{1}\right) \cos \left(\theta_{2}\right), \\
& H_{x y}=b \cos \left(\theta_{1}\right) \cos \left(\theta_{2}-\alpha\right), \\
& H_{y x}=b^{*} \cos \left(\theta_{1}-\alpha\right) \cos \left(\theta_{2}\right), \\
& H_{y y}=\cos \left(\theta_{1}-\alpha\right) \cos \left(\theta_{2}-\alpha\right),
\end{aligned}
$$




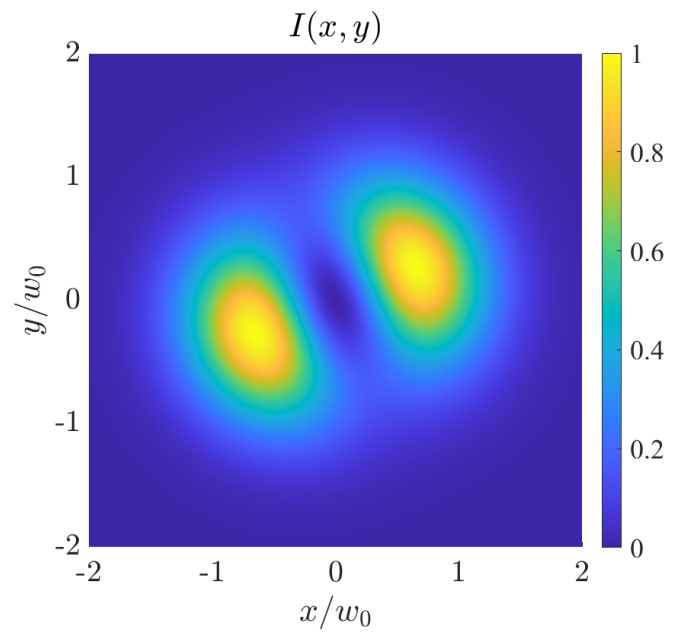

Figure 1. Intensity (normalized to its maximum) at the source plane for the source parameters $\delta=0.25 w_{0}, \alpha=$ $\pi / 4$, and $b=0.5$.

where $b$ is a complex constant satisfying $|b|<1$ and $\alpha$ is a real angle. It can be shown that this kind of source is well defined $[18,19]$.

The evaluation of the CSDM at joint points $\mathbf{r}=$ $\mathbf{r}_{\mathbf{1}}=\mathbf{r}_{\mathbf{2}}$ yields the polarization matrix [20], which describes all characteristics of the source, such as its intensity and the degree of polarization (DOP) at a given position. In particular, it can be easily seen that, for the chosen CSDM, the intensity profile does not present circular symmetry, except for specific values of the source parameters, but that the intensity always vanishes at the source center. This fact can be appreciated in Fig. 1 where a particular choice of the source parameters has been made.

Furthermore, the DOP turns out to be not uniform across the source plane as it can be seen in Fig. 2. The DOP is the same along any selected radial line, but varies from a minimum value that depends on the $b$ value to 1 when varying the angular coordinate.

Several parameters have been introduced to describe the coherence properties of a vectorial source [21-25]. Among them, we will consider the ones proposed in [22-24].

The first one, known as the electromagnetic degree of coherence, gives a measure of the correlation among all components of the fields at the two points as defined in [22]. Fig. 3 shows the behaviour with the angular coordinate of the electromagnetic degree of coherence [22] calculated between two points located on the same circle. The second point is fixed at the angular coordinate $\theta_{2}$. It can be demonstrated that the electromagnetic degree of coherence depends on the angular variables through the square of cosine functions, so it shows a periodic behaviour with $\pi$ period. It is worth noting that this behaviour is different from that observed for scalar pseudo Schellmodel beams where the degree of coherence present a circular symmetry $[7,8]$.

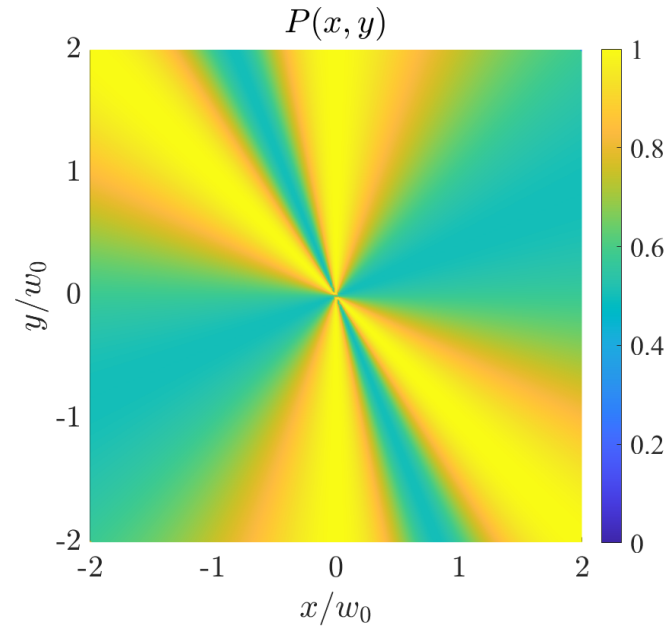

Figure 2. Degree of polarization at the source plane for the same source parameters than those in Fig. $1(\delta=$ $0.25 w_{0}, \alpha=\pi / 4$, and $\left.b=0.5\right)$.

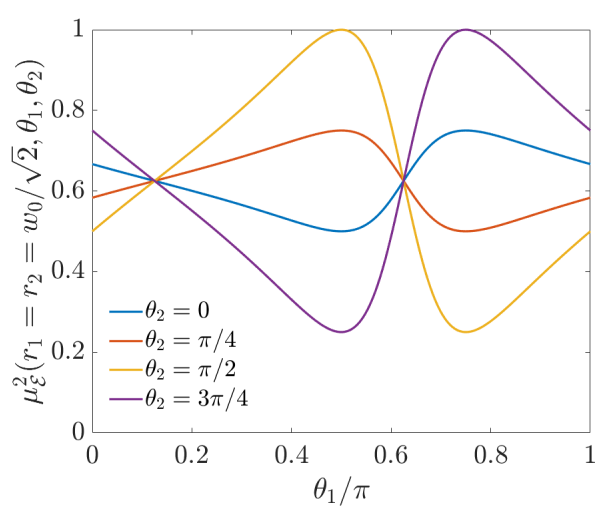

Figure 3. Angular dependence $\left(\theta_{1}\right)$ of the electromagnetic degree of coherence [22] relative to points located on the same circle but at different angular coordinates $\theta_{2}$. The source parameters are the same as in Fig. 1 $\left(\delta=0.25 w_{0}, \alpha=\pi / 4\right.$, and $\left.b=0.5\right)$.

The radial dependence of the electromagnetic degree of coherence [22] shows a maximum for points on the same circle but this maximum has not to be necessarily 1 , even though this parameter is calculated for the field at the same point (see Fig. 4). This parameter decreases from such a maximum value in a Gaussian way when one of the two considered points apart from the other in radial direction either towards the center of the soure or to infinity. Similar behavior is observed if the two points are located on different radial lines, the only difference is that the maximum value changes.

The second analyzed parameter is related to the maximum fringe visibility obtainable in a Young experiment on modifying the interfering fields by means of local unitary transformations (i.e., by means of deterministic nonabsorbing anisotropic optical elements). The behaviour of this parameter along a cir- 

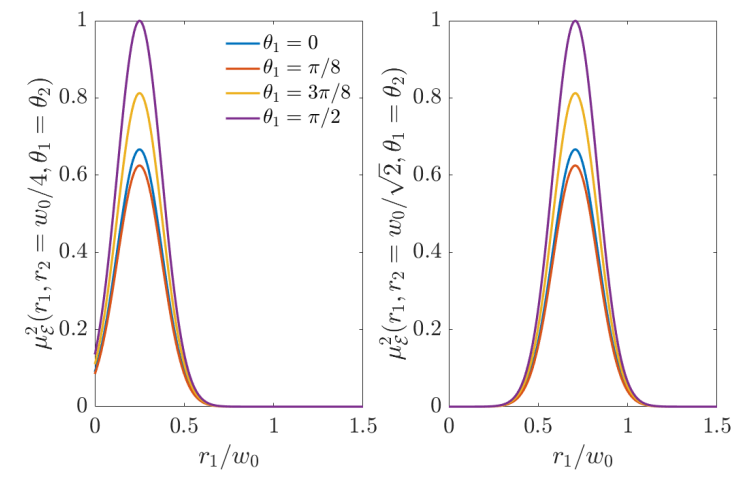

Figure 4. Radial dependence of the electromagnetic degree of coherence [22] relative to a point located at a distance from the source center $r_{2}=w_{0} / 4$ (left) or $r_{2}=w_{0} / \sqrt{2}$ (right) and on the several radial lines forming $\theta_{1}=\theta_{2}$ angle with $x$-axis. The source parameters are the same as in Fig. $1\left(\delta=0.25 w_{0}, \alpha=\pi / 4\right.$, and $\left.b=0.5\right)$.

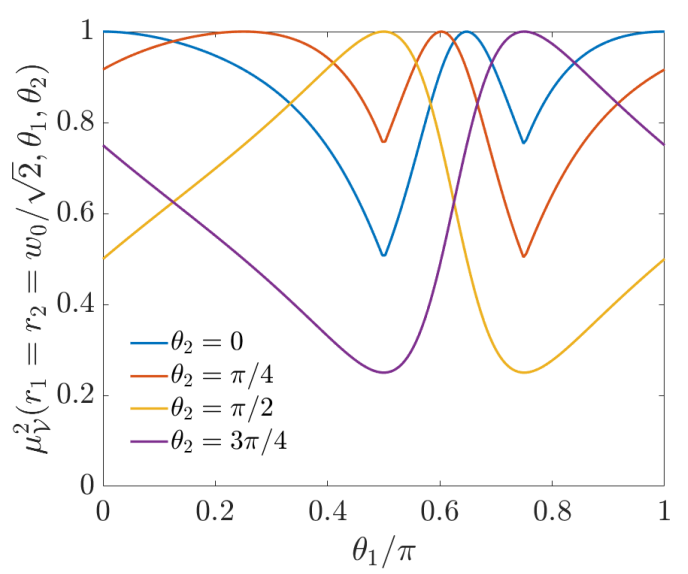

Figure 5. Angular dependence $\left(\theta_{1}\right)$ of the maximum fringe visibility in a Young experiment $[23,24]$ relative to points located on the same circle but at different angular coordinates $\theta_{2}$. The source parameters are the same as in Fig. $1\left(\delta=0.25 w_{0}, \alpha=\pi / 4\right.$, and $\left.b=0.5\right)$.

cle can be observed in Fig. 5. Again, due to the way the source is defined, this parameter shows a peridodic behaviour with the angle variation of one of the considered points with $\pi$ period. Note that a maximum value of 1 (maximum visivility ain a Young experiment) is always reached when the two points coincide and when they are diametrically opposite (on the same circle).

\section{Paraxial propagation}

The Fresnel diffraction integral can be used to obtain the CSDM of the field radiated from the source under paraxial approximation. It can be shown that the CSDM of the propagated beam presents the same angular dependence than that at the source plane, that is, Eq. (1) holds just replacing $W_{P S}$. Then, the polarization properties of the beam remain invariant under propagation and the degree of polarization has the same pattern as in Fig. 2.

The intensity profile, in general changes in propagation, but it is always zero at the axis of the beam. Figure 6 shows the evolution of the intensity distribution with propagation distance. The same parameters as in Fig. 1 are chosen. It can be observed that the maximum intensity is not reached at the source plane (see the colorbar scale) but after a certain propagation distance.

\section{Conclusions}

In summary, a new class of partially coherent, partially and nonuniformly polarized source of the pseudo-Schell model type is proposed and analyzed in detail. The generated fields not only have a structured coherence but also exhibit a non-uniform polarization. The pattern of the degree of polarization remains invariant in propagation. Although the field radiated by these sources does not present circular symmetry, it does present a null in the center for all $\mathrm{z}=$ constant plane. They also have a self-focusing effect. These properties could be very useful in various applications such as particle trapping.

\section{Acknowledgements}

This work has been supported by Spanish Ministerio de Economía y Competitividad under project PID2019 104268GB-C21.

\section{References}

[1] H. Lajunen, T. Saastamoinen, Opt. Lett. 36, 4104 (2011)

[2] Y. Cai, Y. Chen, F. Wang, J. Opt. Soc. Am. A 31, 2083 (2014)

[3] Y. Cai, Y. Chen, J. Yu, X. Liu, L. Liu, Progress in Optics 62, 157 (2017)

[4] M. Santarsiero, R. Martínez-Herrero, D. Maluenda, J.C.G. de Sande, G. Piquero, F. Gori, Opt. Lett. 42, 1512 (2017)

[5] M. Santarsiero, R. Martínez-Herrero, D. Maluenda, J.C.G. de Sande, G. Piquero, F. Gori, Opt. Lett. 42, 4115 (2017)

[6] G. Piquero, M. Santarsiero, R. Martínez-Herrero, J.C.G. de Sande, M. Alonzo, F. Gori, Opt. Lett. 43, 2376 (2018)

[7] J.C.G. de Sande, R. Martínez-Herrero, G. Piquero, M. Santarsiero, F. Gori, Opt. Express 27, 3963 (2019)

[8] R. Martínez-Herrero, G. Piquero, J.C.G. de Sande, M. Santarsiero, F. Gori, Applied Sciences 9 (2019)

[9] J.C. Ricklin, F.M. Davidson, J. Opt. Soc. Am. A 19, 1794 (2002)

[10] O. Korotkova, L.C. Andrews, R.L. Phillips, Optical Engineering 43, 330 (2004) 

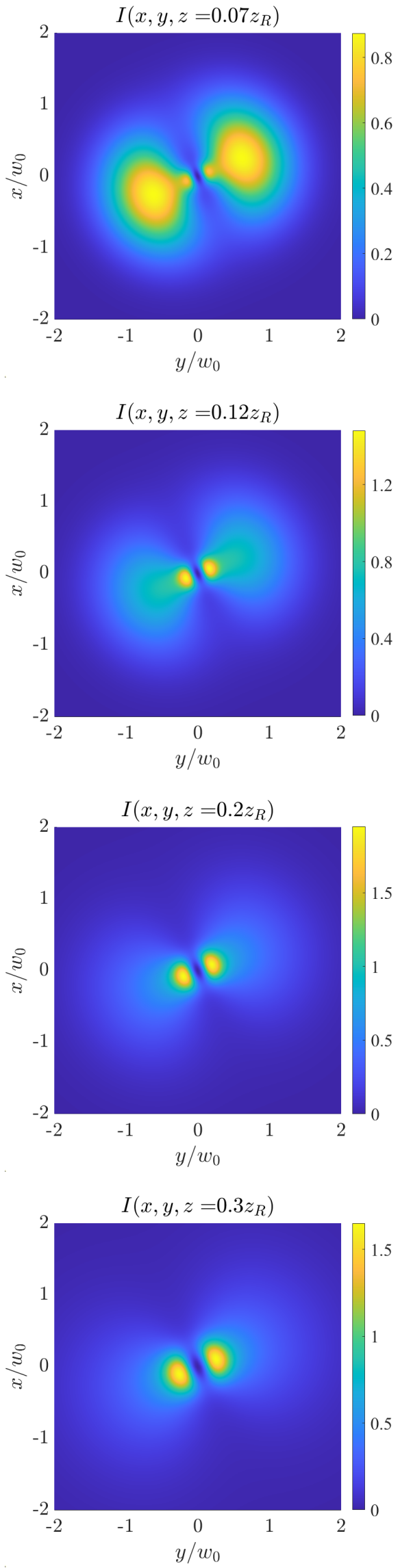

Figure 6. Intensity maps (normalized to the maximum of the intensity at the source plane) for several propagation distances, being $z_{R}=k w_{0}^{2} / 2$ and $k$ the wavenumber. The source parameters are the same as in Fig. $1\left(\delta=0.25 w_{0}\right.$, $\alpha=\pi / 4$, and $b=0.5)$
[11] S.B. Raghunathan, T. van Dijk, E.J.G. Peterman, T.D. Visser, Opt. Lett. 35, 4166 (2010)

[12] C. Zhao, Y. Cai, Opt. Lett. 36, 2251 (2011)

[13] G. Wu, Y. Cai, Opt. Lett. 36, 1939 (2011)

[14] G. Gbur, J. Opt. Soc. Am. A 31, 2038 (2014)

[15] C. Ding, M. Koivurova, J. Turunen, L. Pan, J. Opt. Soc. Am. A 34, 1441 (2017)

[16] M.W.H. IV, Optics 1, 97 (2020)

[17] J.C.G. de Sande, M. Santarsiero, G. Piquero, Optics and Lasers in Engineering 91, 97 (2017)

[18] F. Gori, V. Ramírez-Sánchez, M. Santarsiero, T. Shirai, Journal of Optics A: Pure and Applied Optics 11, 085706 (2009)

[19] R. Martínez-Herrero, P.M. Mejías, Opt. Lett. 34, 2303 (2009)

[20] L. Mandel, E. Wolf, Optical Coherence and Quantum Optics (Cambridge University Press, 1995), ISBN 9780521417112

[21] E. Wolf, Introduction to the Theory of Coherence and Polarization of Light (Cambridge University Press, 2007), ISBN 9780521822114

[22] J. Tervo, T. Setälä, A.T. Friberg, Opt. Express 11, 1137 (2003)

[23] F. Gori, M. Santarsiero, R. Borghi, Opt. Lett. 32, 588 (2007)

[24] R. Martínez-Herrero, P.M. Mejías, Opt. Lett. 32, 1471 (2007)

[25] A. Luis, J. Opt. Soc. Am. A 24, 1063 (2007) 